\title{
Pain associated with specific anxiety and depressive disorders in a nationally representative population sample
}

\author{
Katja Beesdo, Frank Jacobi, Jürgen Hoyer, Nancy C. P. Low, Michael Höfler, Hans-Ulrich \\ Wittchen
}

\begin{abstract}
Objective To examine in a nationally representative sample (a) the differential association of specific anxiety and depressive disorders defined according to DSM-IV with pain disorder (PD) and pain symptoms, and (b) whether pain-associated anxiety and depressive disorders and their comorbidity have different implications in terms of impairment, disability, health care utilization, and substance use.

Method A nationally representative community study was conducted in Germany. Symptoms, syndromes and diagnoses of mental disorders, and pain were assessed in $\mathrm{N}=4,181$ participants aged 18-65 years using the DSMIV/ M-CIDI.

Results Logistic regressions revealed that pain is associated with both specific anxiety and depressive disorders, with increasing significant odds ratios (OR) for medically explained pain symptoms (EPS; OR range: 1.9-2.0), to unexplained pain symptoms (UPS; OR range: 2.4-7.3), to PD (OR range: 3.314.8). PD and UPS persistently showed associations after adjusting for comorbid other anxiety and depressive disorders and physical illnesses. All types of pain, particularly PD/UPS, are associated with decreased quality of life, greater impairment in role functioning, disability, health care utilization, and substance use. Depressive disorders, even more so anxiety disorders and their comorbidity account for a substantial proportion of variance in these functional correlates.

Conclusions Pain is strongly associated with specific anxiety and depressive disorders. In light of the individual and societal burden due to pain, and the demonstrated role of comorbid anxiety or/and depression, our results call for further investigation of the underlying mechanisms for this association as well as targeted treatments for these comorbidities.
\end{abstract}

Keywords Pain, Anxiety disorder, Depressive disorder, Epidemiology, Comorbidity

\section{Introduction}

Pain, anxiety, and depressive disorders are prevalent and burdensome conditions in the community [1, 2, 24, 33, 34, 39, 58, 66, 97]. The intimate and reciprocal relationships between these conditions have been well documented on a symptomatic (dimensional) level $[22,25,27,34,60,72,76,82,88,92]$, as were the clinical-therapeutic implications of these relationships for the management of pain syndromes [9, 38, 52, 85]. The nature and the implications of such complex associations on the diagnostic level, however, remain unclear and understudied. Few studies have systematically examined the relationships between pain conditions and specific well-defined DSM-diagnoses [3, 4], especially in non-clinical, representative population samples, with most research attention given to depressive disorders [18, 21, 33, 52, 59, 69]. Depression was also shown to have a significant impact on disability in pain $[18,20]$. Regarding anxiety disorders, recent findings from population-based samples also indicate associations with pain which seem to be of equal or greater strength compared to the pain-depression associations [21, 26, 33, 53, 58, 59, 91]. To our knowledge, no population-based study has systematically examined the impact of anxiety disorder comorbidity on disability in pain.

Pain as defined by the International Association for the Study of Pain is „,an unpleasant sensory and emotional experience associated with actual or potential tissue damage or 
described in terms of such damage“ [61]. That is, the subjective experience of pain including sensory, emotional, behavioural, and cognitive components is not solely due to tissue damage. Many pain symptoms remain „medically unexplained“ or „incompletely explained“, i.e., the pain cannot be (fully) explained by the presence of general medical condition, by the direct effect of an exogenous substance, or by a mental disorder such as anxiety or depression. According to a review by Kroenke [48], in the health care system at least one-third of somatic symptoms are medically unexplained. There has been extensive debate about the terminology, conceptualization, and classification of such medically unexplained conditions [35, 49, 56, 75, 84, 86]. While in medical routine care, the term „functional syndrome“ is frequently used, the DSMIV [5] and ICD-10 [95] classify pain in which psychological factors are judged to have an important role in the onset, severity, exacerbation, or maintenance of pain as a (somatoform) „pain disorder“. Along with explicit descriptive criteria, this category was meant to increase the reliability of assessment, although it is important to note that this description remains controversial. The categorization of symptoms to be „medically unexplained/somatoform“ can be difficult [49] and theoretically and practically problematic $[56,75]$. This has led some $[49,56,75]$ to suggest simplifying the categorization of pain disorder and to use a more appropriate, ,etiologically neutral“ terminology for physical symptoms including pain. Others [35], however, have underlined the progress and importance of research with somatoform diagnoses as mental disorders integrating biological, psychological, and social aspects.

Acknowledging the controversy surrounding the conceptualization and terminology of „medically unexplained“ pain and „pain disorder“, this paper examines in a large German general population sample the differential association of specific anxiety and depressive disorders defined according to DSM-IV with pain disorder and pain symptoms. Other than a previous report [26], we consider self-reported lifetime medically unexplained and explained pain symptoms. Moreover, we will explore whether the pain-associated anxiety and depressive disorders and their comorbidity have different implications in terms of impairment, disability, health-care utilization, and substance use.

\section{Methods}

The data presented in this paper come from the German National Health Interview and Examination Survey, Mental Health Supplement (GHS-MHS) conducted in 1998/1999. The survey was approved by the institutional review board of the Robert Koch Institute (Berlin, Germany). All participants provided written informed consent. The aims, design, and methods of the survey have been described in more detail elsewhere [40].

\section{Sample}

The GHS (core survey) covered a range of medical and social assessments in a multistage, stratified, cross-sectional, random sample of the general German population aged 18-79 years, drawn from population registries ( $\mathrm{N}=7,124$; response rate: $61.5 \%$ ). The MHS (one of several supplements) included a subsample thereof $(\mathrm{N}=4,181$; conditional response rate: 87.6\%). This subsample can be regarded as representative for the German noninstitutionalized adult population in the age-range of 18-65 years [40]. Older subjects were excluded from the MHS due to unsatisfactory psychometric properties of the used diagnostic assessment instrument - the Munich Composite International Diagnostic Interview (DIA$\mathrm{X} / \mathrm{M}-\mathrm{CIDI}$ ) - in older populations [47]. 


\section{Assessment of mental disorders}

The assessment of mental disorders was based on the computer-assisted version (CAPI) of the Munich Composite International Diagnostic Interview (DIA-X/M-CIDI) [99, 101], a modified version of the World Health Organization (WHO) CIDI, version 1.2, supplemented by questions to cover DSM-IV [4] and ICD-10 criteria [95]. The DIA-X/M-CIDI was administered by clinically trained interviewers (psychologists and MDs). Psychometric properties of the DIA-X/M-CIDI were found to range from acceptable to very good for all diagnoses considered in the current study: the test-retest reliability ranged from kappa $=0.45$ for GAD to 1.00 for panic disorder [99]. Inconsistencies in GAD were mainly due to different responses in regard to the 6-month time duration; retest-reliability of the GAD stem question, however, was good and in the range of other disorders (kappa $=0.70)$. Validity of the DSMIV/M-CIDI diagnoses compared to independent clinical consensus diagnoses by treating physicians was estimated with kappa ranging from 0.50 for somatoform disorder to 0.96 for major depressive episode (MDE) [70]. Age-of-onset reliability estimates are also established with intra class coefficients ranging from 0.45 for specific phobia environmental-subtype to 0.97 for GAD and MDE [99, 100].

Pain disorder The diagnosis of (somatoform) pain disorder was generated by using the standard DSM-IV/MCIDI algorithms [101]. In the somatoform disorders section of the DIA$\mathrm{X} / \mathrm{M}$-CIDI, the presence of the following painful conditions is initially assessed from the respondents on a lifetime basis: abdominal pain, back pain, joint pain, limb pain, chest pain, headache, excessively painful menstruation, rectal and genital pain, and other pain. For commonly experienced pain types (belly pain, back pain, headache, and painful menstruation) the threshold for positive endorsement was increased by asking whether the respondent has ever had „a lot of trouble“ with this pain. Whenever the respondent acknowledged having experienced at least one of these pain types, the interviewer enters a standardized complex set of probe questions (see Fig. 1) to evaluate the nature of the complaint [73, 96].

The probe questions start with establishing clinical severity/significance. A symptom is considered „clinically significant“ if the symptom prompts help-seeking from a medical doctor or another health professional, the use of medication more than once for it, or if the symptom interfered a lot with life or activities (criterion A). For headaches and painful menstruation, positive endorsement of medication use more than once was not considered as sufficient for establishing clinical significance. Here, respondents must have additionally reported medication prescription by a doctor (pertaining to headaches and painful menstruation), or the use of prescription-free medication at least three times a week (pertaining to headaches) [101].

Next, further probe questions establish from the respondents whether there are any physical or substancerelated factors that explain the occurrence of the pain symptom (,medically explained“ pain). As shown in Fig. 1, there are several paths with various questions to be answered before a symptom is considered to be „medically unexplained“ (somatoform). Only when the symptom, per respondents' report, was not or not always explained by medical diagnosis (e.g., doctor's diagnosis of migraine for headaches) or injury, or the use of medication, drugs or alcohol, the symptom is rated as „medically unexplained“ and consistent with the DSM-IV stipulation - counted towards a diagnosis of somatoform pain disorder (criterion C; note that the controversial 'psychological factors' criterion [35, 49, 56, 
$75,84,86]$ is particularly difficult to assess with a standardized instrument in an epidemiological study [compare 30]).

If at least one painful condition was found to be present as clinically significant and medically unexplained (somatoform), further questions were asked to assess impairment and distress (DSM criterion B) as follows [4]: „Did the pain keep you from working or seeing friends or relatives for 6 months or more?“, „How much did the pain interfere with your life and daily activities?" If the respondent acknowledged either impairment OR a lot interference with life, then the DSM-IV diagnosis of pain disorder was given.

DSM criterion D (the symptom is not intentionally produced or feigned) was not assessed by the DIA-X/M-CIDI. The exclusion criterion $\mathrm{E}$ (the pain is not better accounted for by a mood, anxiety, or psychotic disorder) will be considered by providing the proportion of respondents who reported that their physician attributed their pain to ,anxiety, panic attacks, or depression“.

Keeping the controversy regarding somatoform pain and pain disorder in mind, we consider in the current paper the diagnosis of (somatoform) pain disorder (PD) as based on respondents' self-reports and DIA-X/M-CIDI algorithms. In addition, we included the following pain categories below this diagnostic threshold: (a) at least one clinically significant, unexplained (somatoform) pain symptom (UPS) but no PD and (b) at least one clinically significant, explained (non-somatoform) pain symptom (EPS) but no unexplained (somatoform) pain symptoms. Individuals with no clinically significant pain symptoms (no PS; includes individuals without any pain and individuals with non-significant pain) were used as reference group.

Throughout our paper, we report on self-reported lifetime occurrence of pain, because the age-of-recency of pain - and therefore 12-month status - was only assessed among individuals with any medically unexplained (somatoform) pain.

Depressive and anxiety disorders Diagnoses of depressive disorders (major depression and dysthymia) and anxiety disorders (specific phobia, social phobia, agoraphobia, phobia not otherwise specified (NOS), generalized anxiety disorder (GAD), panic disorder, and obsessive compulsive disorder (OCD) were also assessed using the DIA-X/M-CIDI and the DSM-IV algorithms [101]. Because lifetime information is not available for all the anxiety disorders, we refer to 12-month anxiety and depressive disorders throughout the paper.

Substance use and substance use disorders Substance use and substance use disorders were also assessed using the DIA-X/M-CIDI. In the current paper, the following dichotomous variables for substance use were considered for analyses on correlates: during the past year, (a) harmful alcohol use (weekly alcohol use, 20/40 g alcohol per drinking day for women/men), (b) daily smoking over a period of at least 4 weeks, and (c) any illicit medication/ substance use (including: cannabis, stimulants, sedatives/ hypnotics/anxiolytics, opiates, cocaine, PCP, psychodelics, inhalants, „other“ substances).

\section{Assessment of somatic disorders}

A self-report questionnaire was used to asses the presence of 44 physical diagnoses from 16 disease groups (e.g., cardiac diseases, diabetes, cancer, allergies (see [40]). Taking this information into account, study doctors conducted a computer-assisted standardized interview and established lifetime, 12-month and point prevalences of physical disorders. In this paper, 
we use a variable containing the number of physical disorders during the past 12 months (range 0-9) as control variable.

\section{Other measures}

Assessment of health related quality of life The German version [14, 94] of the well-validated self-report Medical Outcome Study 36-item short form (SF-36) [13, 15, 23, 37, 57, 83] was used to assess eight health concepts for the past 30 days. Principal component analysis has identified two dimensions of the SF-36: a physical component score and a mental component score [93]. These scores were standardized to $\mathrm{M}=50$ and $\mathrm{SD}=10$.

Assessment of disability Similar to previous surveys [e.g., 74], past 4-week disability was examined by the selfreported number of days being unable to carry out usual daily activities (school, study, work, household) (1) due to mental (emotional, psychosomatic, or psychiatric) problems, (2) due to alcohol, drug or medication use, and (3) due to physical problems and illnesses. The number of overall disability days was generated by counting a full day for each day a person was totally unable to carry out usual activities and adding a half-day for each day a person was partly disabled to carry out usual activities. This summary variable reflects the number of disability days in the past month ranging from 0 to 28 days (higher summary scores were set to 28 days).

Assessment of health care utilization The assessment of health care utilization included selfreported data on the number of (1) general practice visits, (2) consultations with specialists (12 domains, including neurologists, psychiatrists, and psychologists), and (3) days spent in hospital within the past 12 months.

\section{Statistical analyses}

Statistical analyses were done with the STATA software package, release 10.0 [81]. Data (\%, $\mathrm{M}$, SD, and regressions/ ratios) were weighted for age, gender, region and screening status in order to address different sampling probabilities and systematic non-response [40]; number of cases is reported unweighted $(\mathrm{N}, \mathrm{n})$.

Logistic regression analyses were used to quantify associations [odds ratios, OR; with 95\% confidence intervals (CI), and P values] between PD, UPS, and EPS on the one hand and specific depressive disorders and anxiety disorders on the other (crude association, controlled for age and gender only). To test whether those associations persist even after adjusting for comorbid conditions, first anxiety and depressive disorders were included as control variables in the regressions, and then additionally a variable containing the number of physical disorders.

Correlates of pain (health related quality of life, disability days, health care utilization and substance use) were also assessed by regression analyses. For dichotomous outcomes (i.e., substance use), associations were assessed with odds ratios (ORs) from logistic regressions. Associations with count variables (i.e., number of disability days, number of health care visits) were assessed with mean ratios (MRs) from negative binomial regressions [50]. These regressions are useful for positively skewed distributions of infrequent events and the associated mean ratios reflect the change in the expected count of a particular event per unit increase in the covariate. Mean ratios from gamma regressions were used for gammadistributed SF-36 outcomes. A significant ratio of $<1$ indicates a decrease of health related quality of life. First, associations were assessed controlling for age and gender only. In order 
to estimate the degree to which comorbid anxiety or/and depressive disorders account for these associations, the analyses were rerun while additionally controlling for (a) anxiety disorders, (b) depressive disorders, and (c) anxiety and depressive disorders. The relative change in association between pain and predictors was then calculated. ${ }^{1}$ The results reflect the percentage change in the association between pain and the correlates due to adjustment of anxiety (A), depressive (D), or anxiety and depressive disorders (A + D). Negative percentages reflect smaller increase in disability, health care utilization and substance use, while positive percentages reflect smaller decrease in health related quality of life.

\section{Results}

Prevalence and comorbidity of pain, anxiety disorders and depressive disorders

Four out of five individuals (81.2\%) in the general population experienced at least one significant pain symptom in their lifetime (Fig. 2). The majority of affected individuals had EPS only (55.2\%), with the remaining reporting at least one UPS (44.8\%). Among those with UPS, one-third met criteria for PD (35.1\%). Back pain (40.5\%), joint pain (32.5\%) and abdominal pain (16.1\%) were the three most commonly experienced significant explained pain types; among unexplained pain symptoms headache (12.7\%), back pain (8.5\%), and abdominal pain (8.3\%) were reported most frequently.

Overall, lifetime prevalence rates for mutually exclusive diagnostic pain categories were 12.7\% for PD, $23.7 \%$ for UPS (no PD), and 44.8\% for EPS (no UPS/PD). The mean number of unexplained pain symptoms was $1.7(\mathrm{SD}=1.0$, range $1-7)$ in the $\mathrm{PD}$ group and $1.5(\mathrm{SD}=$ 0.8 , range: $1-6$ ) in the UPS group; the mean number of explained pain symptoms among individuals with PD, UPS, and EPS were $1.8(\mathrm{SD}=1.6$, range $0-7), 1.4(\mathrm{SD}=1.3$, range: range $0-6)$, and $2.0(\mathrm{SD}=1.2$, range $1-8)$, respectively. The proportion of individuals reporting unexplained pain symptoms only (no significant explained pain symptoms) was $20.3 \%$ in the PD and $30.2 \%$ in the UPS group. Of note, $20.0 \%$ of the PD and $20.9 \%$ of the UPS cases who had consulted their physician for at least one of their pain symptoms $(n=532$ of the PD and $n=952$ of the UPS cases) reported that their pain was specifically attributed to nerves or stress by their physician (compare Fig. 1). In contrast, only a few cases (PD: $n=4$, $0.7 \%$; UPS: $n=6,0.6 \%$ ) stated that their physician explained their pain by anxiety, panic, or depression.

Gender and age differences in lifetime prevalence rates of the pain categories are shown in Fig. 3a. Compared to men, women significantly more frequently had PD (females: $17.8 \%$ vs. males: 7.8\%, OR = 2.6, 95\% CI: 2.1-3.2, P < 0.001) and UPS (females: 27.8\% vs. males: $19.6 \%$, OR $=1.6,95 \%$ CI: $1.3-1.9, \mathrm{P}<0.001$ ), but less frequently EPS (females: $29.3 \%$ vs. males $50.3 \%$, OR $=0.6,95 \% \mathrm{CI}$ : $0.5-0.7, \mathrm{P}<0.001)$. The lifetime prevalence of EPS is higher in older age groups both in males (35-49 vs. $18-34$ years: OR $=1.4$, 95\% CI: $1.1-1.8$, $\mathrm{P}=0.006$; $50-65$ vs. $18-34$ years: $\mathrm{OR}=1.9,95 \% \mathrm{CI}: 1.5-2.5, \mathrm{P}<0.001)$ and in females (3549 vs. $18-34$ years: $\mathrm{OR}=1.4,95 \% \mathrm{CI}: 1.1-1.7, \mathrm{P}=0.008$; $50-65$ vs. $18-34$ years: $\mathrm{OR}=1.8$, 95\% CI: 1.4-2.2, P < 0.001). In contrast, such increases in lifetime prevalence with higher age are not found for PD and UPS (all comparisons not significant with the exception of a higher rate for $\mathrm{PD}$ in 50-65 vs. 18-34 years old males: $\mathrm{OR}=1.8,95 \% \mathrm{CI}$ : $1.2-2.8, \mathrm{P}=0.008$ ).

\footnotetext{
${ }^{1}$ For ratios $>1$ : $\{0$ - [1 - (adjusted ratio - 1) / (unadjusted ratio - 1)] x 100\}, For ratios $<1$ : $\{1$ - [adjusted ratio - 1) / (unadjusted ratio - 1)] x 100\}.
} 
The 12-month prevalence rates for any anxiety disorder and any depressive disorder were 14.5 and $10.9 \%$, respectively. Age and gender-specific rates are lower, but similar to the ones of unexplained pain (Fig. 3b). Relative to males, females more frequently reported an anxiety disorder (males: 9.2\%, females: 19.8\%; OR $=2.4,95 \% \mathrm{CI}: 2.0-2.9, \mathrm{P}<0.001$ ) and a depressive disorder (males: 7.6\%, females: 14.2\%; OR = 2.0, 95\% CI: 1.6-2.5, P < 0.001). Similarly, age was unrelated to anxiety disorders in both genders and to depressive disorders in males; females of higher age groups had higher rates of depression (35-49 vs. 18-34 years: $\mathrm{OR}=1.4,95 \%$ CI: $1.03-1.9, \mathrm{P}=0.034 ; 50-65$ vs. $18-34$ years: $\mathrm{OR}=1.4,95 \% \mathrm{CI}: 1.02-2.0$, $\mathrm{P}=0.035)$.

As also shown in Fig. 2, individuals with lifetime PD are frequently comorbid with anxiety (31.7\%) and depressive (24.5\%) disorders in the prior 12-months. Comorbidity with anxiety and depressive disorders decreases when the definition of pain is broadened to any unexplained pain (anxiety disorder: 24.2\%; depressive disorder: $17.8 \%$ ) and to any significant pain (anxiety disorder: 16.5\%; depressive disorder: 12.2\%).

Overall, $1.9 \%$ of the sample reported all three diagnostic categories: lifetime PD as well as 12-month anxiety, and depressive disorders. This prevalence increases to 3.5 and $4.7 \%$ when broadening the pain definition to any unexplained pain and any significant pain, respectively.

\section{Associations between pain and specific anxiety and depressive disorders}

Using the mutually diagnostic pain categories, consistently higher proportions of 12-month rates of specific anxiety and depression diagnoses are found among individuals with PD and UPS in comparison to the proportion in the total sample; those without significant pain or EPS revealed consistently lower rates (Table 1). Of note, all individuals with GAD reported any significant pain with the majority suffering from PD.

Table 2 shows the respective pain associations for the specific depressive and anxiety disorders. Starting with a crude model (controlling for age and gender only, upper part of Table 2) and using individuals without significant pain as reference, it was observed that not only PD (OR range: 3.3-14.8) but also UPS (OR range: 2.4-7.3) were associated with all of the specific depressive and anxiety disorders (exception: OR not significant for UPS and OCD). Odds ratios for EPS were consistently lower and only few were significant (dysthymia, specific phobia, phobia NOS, any anxiety disorder). Using EPS as comparison group, significantly stronger relationships to almost all of the anxiety and depressive disorders are found for PD and UPS, respectively. Overall, PD is most strongly associated with anxiety and depressive disorders as also indicated by significantly greater odds ratios relative to UPS.

We repeated the analyses while additionally controlling for comorbidity between and among depressive and anxiety disorders, i.e., the association for any depressive disorder with pain was controlled for any comorbid anxiety disorder and the association between for example social phobia and pain was controlled for comorbid other anxiety disorders and any comorbid depressive disorder (Table 2, middle part). Although there was a consistent drop in the size of the odds ratios, most associations remained significant. Using no significant pain symptoms as reference group, there were only two attenuated OR in each of the significant pain categories (PD: social phobia and OCD, UPS: social phobia and agoraphobia, EPS: dysthymia and phobia NOS).

Additionally adjusting for the number of physical disorders in a third step (Table 2, lower part), a rather minor drop in the size of the odds ratios was observed. Using no pain as the 
reference, only two more associations were attenuated additionally (PD: agoraphobia, EPS: specific phobia). Relative to EPS, unexplained pain (PD and UPS) still revealed significantly stronger relationships to almost all of the anxiety and depressive disorders.

As individuals with PD and UPS may also experience explained pain symptoms, we finally also controlled for the number of co-occurring EPS. All association remained relatively unchanged with the exception that the associations for PD and UPS with major depression were attenuated when no PS was used as reference group (not shown in table; results available upon request).

In order to investigate reasons for the strong associations between PD and UPS on the one hand and anxiety and depressive disorders on the other hand, we first wanted to rule out artifactual comorbidity due to misclassification. As stated earlier, only very few of the pain cases (PD: $n=4,0.7 \%$; UPS: $n=6,0.6 \%$ ) stated that their physician attributed the pain directly to anxiety, panic, or depression. Further, the examination of the temporal order of onset revealed that only few comorbid cases reported a same year onset of pain and anxiety disorder (PD: $\mathrm{n}=11,6.2 \%$; UPS: $\mathrm{n}=15,6.7 \%$ ), respectively, pain and depressive disorder (PD: $n=10,7.0 \%$; UPS: $n=16,9.1 \%$ ). Pain began at least 1 year prior to depression in the majority of cases (PD: $n=107,72.5 \%$; UPS: $n=105,66.9 \%$ ), whereas the onset of comorbid anxiety disorder was found to be equally frequently prior to (PD: $n=97,46.9 \%$; UPS: $n=94$, $38.3 \%$ ) or after pain onset (PD: $n=94,46.9 \%$; UPS: $n=134,55.0 \%$ ).

A further exploration of the pain-anxiety/depression associations revealed no interaction effects with age (as dimensional measure) and only two interactions with gender. Relative to no significant pain, the associations between UPS and any depressive disorder (interaction with gender: $\mathrm{OR}=3.1$, 95\% CI: 1.3-7.3, $\mathrm{P}=0.011$ ) and major depression (interaction with gender: OR = 3.4, 95\% CI: 1.3-8.9, P = 0.015), respectively, were significantly larger for females than for males.

We also examined whether specific pain sites were particularly associated with any anxiety and/or depressive disorder. There was no consistent indication for this, as almost all explained pain symptoms were associated with any depressive disorder (OR range: 1.3-1.9; exceptions: chest pain, painful menstruation, other pain) and any anxiety disorder (OR range: 1.4-1.8; exceptions: headache, painful menstruation). This lack of specificity was even more pronounced for unexplained pain symptoms with overall higher associations (OR range for any depressive disorder: 1.5-2.8; exception: other pain; OR range for any anxiety disorder: 1.9-4.8; no exceptions).

Finally, we examined whether duration/persistence characteristics were particularly important for the strong associations between unexplained pain and any anxiety and depressive disorder. Among individuals with PD, but not UPS, self-reports of having experienced pain for a period of 6 months, or that their pain bothered most of the time, were indicative of any depressive disorder $(\mathrm{OR}=1.2,95 \% \mathrm{CI}$ : $1.1-1.3, \mathrm{P}=0.001)$ and any anxiety disorder $(\mathrm{OR}=1.2,95 \% \mathrm{CI}$ : $1.1-1.3, \mathrm{P}<0.001)$ relative to individuals with unexplained pain who did not report this. Furthermore, individuals with PD who reported being kept from working or seeing friends/relatives for 6 months or more were also more likely to report any depressive disorder $(\mathrm{OR}=1.2,95 \% \mathrm{CI}: 1.02-1.3, \mathrm{P}=0.023)$ and any anxiety disorder $\mathrm{OR}=1.2,95 \% \mathrm{CI}: 1.1-1.3$, $\mathrm{P}=0.003)$.

\section{Correlates of pain}


Table 3 shows measures of self-reported quality of life, disability, health care utilization, and substance use by pain group and Table 4 contains their respective associations. All pain categories are associated with a decreased physical health-related quality of life, a higher number of disability days, increased general practice and specialist health care visits, and higher rates of illicit medication/substance use. PD and UPS further revealed a decreased mental health-related quality of life and higher rates of harmful alcohol use; PD and EPS groups showed an increased number of hospital days and higher rates of daily smoking. Overall, there is some evidence for dose-response relationships as indicated by increasing ratios from EPS to UPS to PD for most of the correlates.

An exploration into the change of these associations by adjusting for comorbid anxiety or/and depressive disorders revealed that all associations in all of the pain groups were decreased as indicated by ORs approaching 1.0. Percentages for association change were largest for the unexplained pain groups (PD and UPS). Adjusting for anxiety disorders only generally had higher effects compared to depressive disorders only for all examined correlates with the exception of days in hospital; largest effects were found when adjusting for both anxiety and depressive disorders.

\section{Discussion}

Using a large community sample, the present study examined the differential association of specific DSM-IV-defined anxiety and depressive disorders with pain disorder and pain symptoms as assessed via standardized diagnostic instrument. Keeping in mind the ongoing controversy surrounding the pain classification [35, 49, 56, 75, 84, 86] as well as some limitations adjunct to this study (see below), there are two major findings. First, there is a high association between pain and both specific anxiety and depressive disorders, with strong evidence for a dose-response relationship. Second, although all levels of pain are associated with decreased health-related quality of life and increased disability, health care utilization and substance use, such adverse functional outcomes are more likely in UPS/PD. Anxiety and depressive disorders and their comorbidity account for a substantial part of these adverse outcomes, with some indication for differential effects.

Our reported 12-month prevalence rates for anxiety and depressive disorders are in the range of other studies [46, 97]. In terms of pain prevalence, given the lifetime assessment, it was not unexpected that four out of five individuals reported having experienced any significant pain, particularly as our results showed the expected increase in EPS with older age. The lifetime prevalence for unexplained pain was similar across all age groups, indirectly indicating a stable phenomenon. A direct comparison of our pain rates with other studies is difficult due to extensive variance in pain definitions and methods used [e.g., 20, 21, 26, 30, 36, 59, 91]. Our findings indicate that almost half of the individuals with significant lifetime pain report at least one of their pain symptoms not being (fully) explained by physical/substance-related factors (any UPS); overall, 12.7\% of the general population met criteria for PD. This is consistent with prior research that documented high rates of somatoform pain [30, 36, 53]. Together with our findings of decreased health-related quality of life and increased disability, help seeking, and substance use among pain sufferers, these high prevalence rates indicate a substantial public health and societal burden associated with pain in general and PD and UPS in particular.

Our study confirmed the well-known symptomatic relationship between pain and depression and anxiety on the DSM-IV diagnostic level for various specific depressive and anxiety 
disorders. PD and UPS, but not EPS, continued to show strong and consistent associations with specific anxiety and depressive disorders after adjusting for comorbidity with other disorders and physical illnesses. Furthermore, the associations between PD/UPS and specific anxiety and depressive disorders also persisted, when co-occurring EPS were controlled. These findings suggest a prominent role of unexplained pain in the pain-mental disorder association that warrants further research and clinical attention, irrespective of the unknown future of such conditions in terms of labelling and diagnosis [compare: 51]. Our findings also highlight the importance of the anxiety disorder comorbidity in pain. In line with the few available data from community samples [33, 58, 59, 91], the associations between pain and anxiety disorders were of equal or greater strength compared to the pain-depression associations. Of note, despite no overlap in DSM-IV diagnostic criteria, a particularly high (100\%) comorbidity rate was found for GAD and pain. As GAD differs from other anxiety disorders in various respects, this strong association requires further investigation [11].

Noteworthy, in order to rule out artifactual comorbidity we investigated whether physicians attributed the pain explicitly to anxiety or depression, or whether pain and anxiety/depression emerged at the same time. None of these explanations accounted sufficiently for our finding of high associations between unexplained pain and anxiety and depressive disorders.

Consistent with other research [20,91], our study also indicates a strong link between pain and adverse functional outcomes and health care utilization. Some studies [18, 20, 91] suggest that mental disorders such as depression have a significant impact on disability among individuals with pain. Our study adds that comorbid anxiety disorders and comorbid depressive disorders as well as their comorbidity account for a substantial degree of the decreased quality of life, greater impairment in role functioning, disability, health care utilization, and substance use among individuals with pain with some indication for differential effects. First, the comorbidity with anxiety and/or depressive disorders seems to be of greater importance for the adverse correlates in individuals with UPS/PD as compared to EPS. Second, there is some indication for a higher relevance of anxiety disorders than depressive disorders for the adverse pain outcomes, with the exception of days in hospital. Limitations of our study are as follows: first, beyond the general problems associated with assessing and categorizing symptoms as „medically (un)explained“ [49, 56, 75], in the current study the threshold levels for both clinical significance and absence of medical explanation may be perceived as rather low. Second, since no age-of-recency information was available for explained pain symptoms, we considered lifetime occurrences of pain, which may be subject to recall bias. Adjunct to this, the status of lifetime pain categories during the 12month period prior the interview, for which anxiety and depressive disorders have been assessed, remained unclear. Third, due to our epidemiological study method, our diagnostic categories rely on respondents' self-report, and thus their perceptions, rather than on a medical appraisal/evaluation of pain symptoms. The DIA-X/M-CIDI diagnoses pain disorder with imperfect validity.

With these limitations in mind, our results - combined with findings from previous studies have implications for research and practice. Given the high prevalence and association of pain, anxiety and depressive disorders, it is crucial to investigate potential causes for these relationships in order to develop and test targeted interventions. For the comorbidity between pain and depressive disorders, prior research indicates a bi-directional aetiology [52, 78], with some indication, that depression is rather a consequence than an antecedent of pain [9, 25]. For the pain-anxiety disorder comorbidity, not as much systematic research into the temporal order has been performed. Given findings of an earlier incidence of anxiety disorders in comparison to depressive disorders $[39,45,100]$, it is likely that anxiety disorders, more 
frequently than depressive disorders, occur temporally prior to pain. Although limited by the methodological restriction that lifetime information was not available for all the anxiety and depressive disorders in the present study, our explorations into the temporal order of onset are in line with these assumptions: While depressive disorders occurred temporally secondary to pain in the majority of cases, anxiety disorders had their onset about equally frequently prior to and after pain. Our cross-sectional, epidemiological study does not allow for conclusions on causality. Further research is necessary to study possible direct links between pain and anxiety/depression and potential differences in pain aetiology in depression versus anxiety.

Other explanations for the frequent comorbidity of pain and anxiety/depression are perpetuating and/or mediating processes and common underlying aetiological/pathogenic factors. Pain, anxiety disorders, and depressive disorders all reveal associations with similar social and environmental variables (e.g., low socioeconomic status, adverse life experiences) $[10,39,43,89]$. These conditions may also be perpetuated by similar cognitive processes and behavioural factors (e.g., catastrophizing, helplessness, fear of pain, pain/anxiety sensitivity, avoidance and adverse health behaviours) [6, 9, 17, 44, 63, 71, 78, 88, 92], and possibly imply an overlapping genetic and (neuro-) biological (hypothalamic-pituitary-adrenal axis, ascending/descending serotonergic/noradrenergic neurotransmitter pathways) vulnerability, as has been suggested primarily for pain and depression [9, 12, 16, 17, 52, 67, 78, 79, 88]. Given the strong association between pain and anxiety/ depressive disorders and the potential for common underlying mechanisms for those associations, it is conceivable that pain symptoms may even be an integral part of mental disorders as suggested by some authors in regard to depression [e.g., 68, 80]. Our findings indicate that this suggestion may be extended to at least some of the anxiety disorders. However, there is also a body of evidence that antidepressants have an independent mode of action in depression (antidepressant effect) and pain (analgesic effect) $[67,88]$, indirectly suggesting the presence of separate conditions.

From a clinical perspective, our findings suggest that health professionals should add to their awareness of the strong association between pain and a range of anxiety and depressive disorders since these conditions may negatively affect illness course and severity [27, 32, 64, 65], help seeking [10, 20, 29], recognition [8], treatment/referral decisions [42], health care costs [29], and treatment outcome [7, 41]. Patients presenting with pain, anxiety, or depression should be assessed for the presence of other disorders. Although psychological assessment instruments complementing the pain assessment exist [19, 28, 29], patient reports of pain are frequently not followed-up with screening questions for anxiety and depression, resulting in low treatment rates for the emotional disorders [90, 98]. This is unfortunate in light of findings showing that improved depression care is also beneficial for decreasing pain and improving quality of life [54]. Similarly, pain is frequently overlooked in mental health settings and inadequately considered in treatment [68]. Our findings that the pain-anxiety association is at least as strong as the pain-depression association, and that anxiety disorders relative to depressive disorders partly account for a greater proportion of variance in adverse pain outcomes, call for improved awareness and recognition for both types of mental comorbidities. Regarding optimizing treatment, diagnostic versus symptomatic assessment has the advantage of clearer therapeutic implications. Both cognitive-behavioural interventions and pharmacological treatments are indicated for sufferers of pain and anxiety and/or depression [31, 55, 62, 67, 77, 79, 87, 88]. Irrespective of future classification and labelling, particular attention should be given to observations or patient's reports of unexplained pain, as its presence is associated with increased mental comorbidity and negative functional outcomes. 


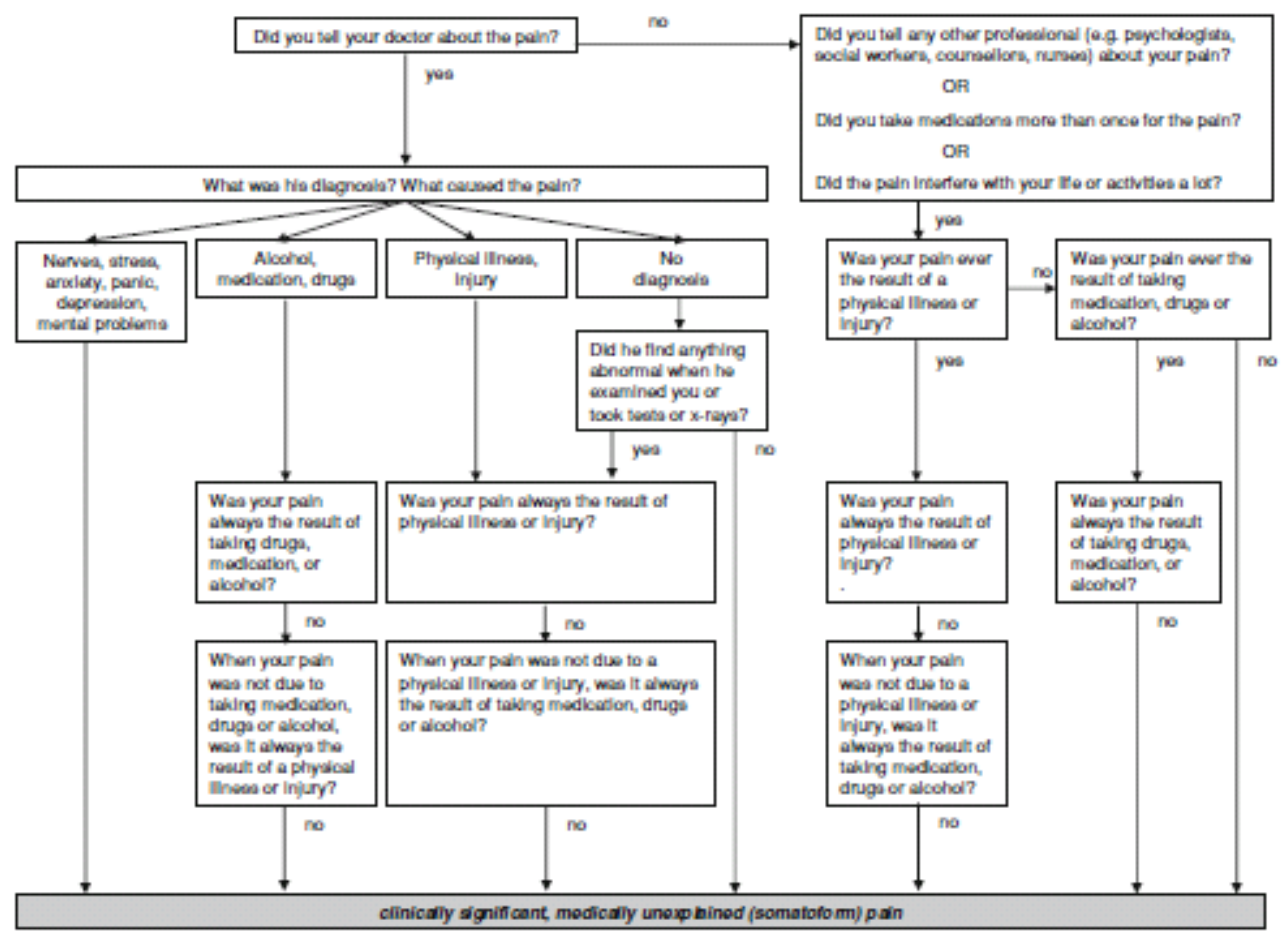

Fig. 1 Structured diagnostic algorithm for the assessment of clinically significant, medically unexplained (soma somatoform disorder section of the DIA-X/M-CIDI

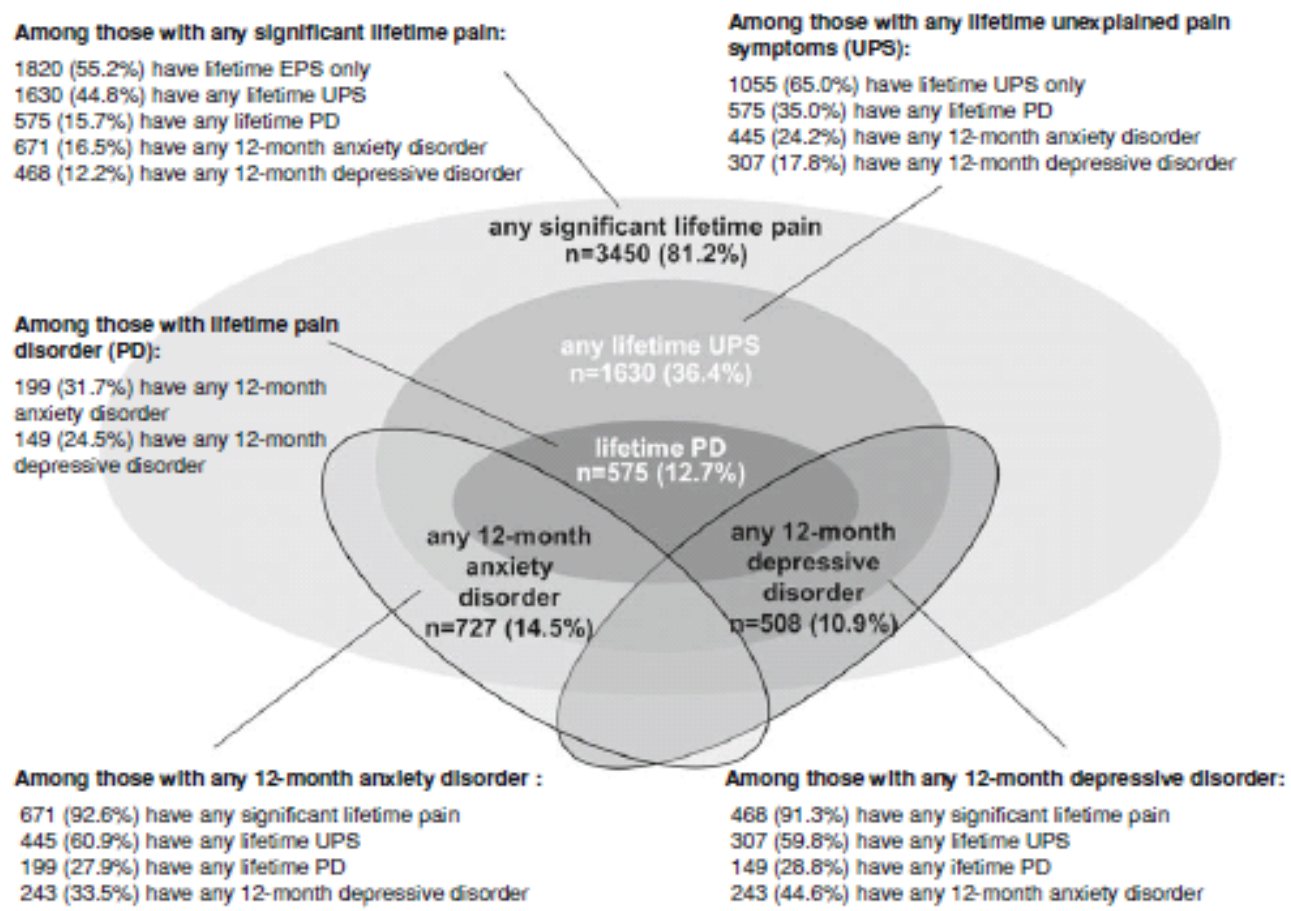

Fig. 2 Prevalence and comorbidity of lifetime pain (not mutually exclusive groups) and 12 -month anxiety and depressive disorders $(N=4,181$ ) EPS explained pain symptoms, UPS unexplained pain symptoms, $P D$ pain disorder 
Fig. 3 Prevalence of lifetime pain (mutually exclusive groups) and 12-month anxiety and depressive disorders by gender and age $(N=4,181)$ EPS explained pain symptoms. UPS unexplained pain symptoms, $P D$ pain disorder
A
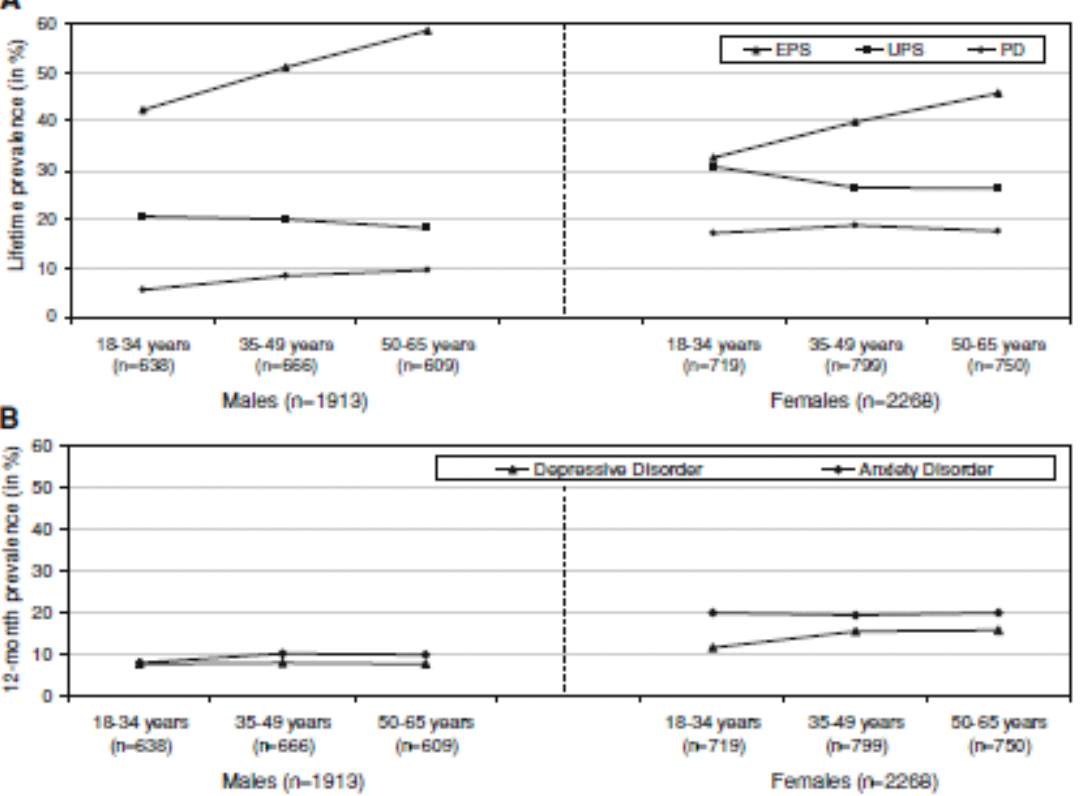

Table 1 Comorbid 12-month depressive and anxiety disorders conditional on lifetime pain (pain groups mutuslly exclusive) $(N=4,181)$

\begin{tabular}{|c|c|c|c|c|c|c|c|c|c|c|}
\hline \multirow[t]{4}{*}{ Mental disorders (12-months) } & \multirow{3}{*}{\multicolumn{2}{|c|}{ Total $(N=4,181)$}} & \multirow{3}{*}{\multicolumn{2}{|c|}{ No PS $(n=731)$}} & \multicolumn{6}{|c|}{ Significant pain (lifetime) } \\
\hline & & & & & \multirow{2}{*}{\multicolumn{2}{|c|}{$\operatorname{EPS}(n=1,820)$}} & \multicolumn{4}{|c|}{ Unexplained pain } \\
\hline & & & & & & & \multicolumn{2}{|c|}{ UPS $(n=1,055)$} & \multicolumn{2}{|c|}{$\mathrm{PD}(n=575)$} \\
\hline & $n$ & $\%$ & $n$ & $\%$ & $n$ & $\%$ & $n$ & $\%$ & $n$ & $\%$ \\
\hline Any depressive discrder & 508 & 10.9 & 40 & 5.0 & 161 & 7.6 & 158 & 14.2 & 149 & 24.5 \\
\hline Major depression & 384 & 8.3 & 32 & 4.3 & 121 & 5.9 & 121 & 10.9 & 110 & 17.9 \\
\hline Dysthymia & 225 & 4.5 & 15 & 1.2 & 60 & 2.6 & 67 & 6.0 & 83 & 13.3 \\
\hline Any anxiety disorder & 727 & 14.5 & 56 & 5.7 & 226 & 10.2 & 246 & 20.1 & 199 & 31.7 \\
\hline Specific phobia & 388 & 7.6 & 29 & 29 & 118 & 5.3 & 136 & 11.1 & 105 & 16.2 \\
\hline Social phobia & 94 & 2.0 & 7 & 0.7 & 28 & 1.5 & 25 & 2.0 & 34 & 5.7 \\
\hline Agoraphobia w/o panic discrder & 105 & 2.0 & 8 & 0.8 & 23 & 1.0 & 36 & 2.6 & 38 & 6.4 \\
\hline Phobia NOS & 173 & 3.4 & 14 & 1.4 & 64 & 2.9 & 55 & 4.7 & 40 & 5.8 \\
\hline GAD & 73 & 15 & 0 & 0.0 & 17 & 0.8 & 26 & 2.2 & 30 & 5.1 \\
\hline Panic disorder w/ or w/o agoraphobia & 121 & 2.3 & 5 & 0.5 & 23 & 1.0 & 44 & 3.7 & 49 & 7.3 \\
\hline$O C D$ & 38 & 0.7 & 2 & 0.3 & 5 & 0.2 & 12 & 1.0 & 19 & 3.0 \\
\hline
\end{tabular}

N/h number unweighted, \% percent weighted, no PS so significant pain symptoms, EPS explained pain symptoms, UPS unexplained pain symptoms, $P D$ pain disorder, NOS not otherwise specified, $G A D$ generalized anxiety disorder, $O C D$ obsessive compulsive disorder 


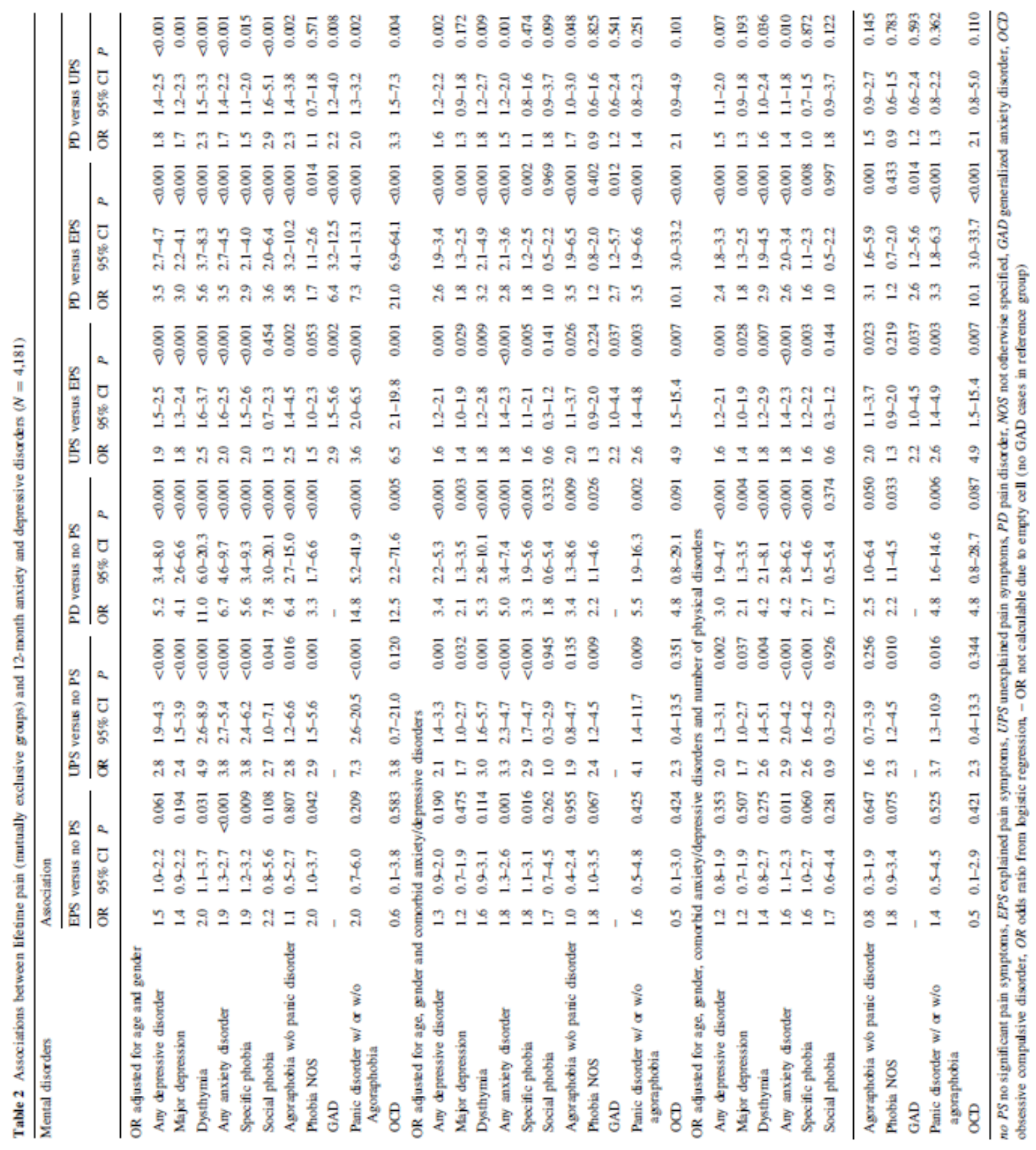


Table 3 Quality of life, disability, health care utilization, and substance use by lifetime pain status (mutually exclusive groups) $(N=4,181)$

\begin{tabular}{|c|c|c|c|c|c|c|c|c|}
\hline \multirow[t]{4}{*}{ Correlates } & \multirow{3}{*}{\multicolumn{2}{|c|}{ No PS $(n=731)$}} & \multicolumn{6}{|c|}{ Clinically significant pain (lifetime) } \\
\hline & & & \multirow{2}{*}{\multicolumn{2}{|c|}{$\operatorname{EPS}(n=1,820)$}} & \multicolumn{4}{|c|}{ Unexplained pain } \\
\hline & & & & & \multicolumn{2}{|c|}{ UPS $(n=1,055)$} & \multicolumn{2}{|c|}{$\mathrm{PD}(n=575)$} \\
\hline & $M$ & SD & $M$ & SD & M & SD & M & SD \\
\hline \multicolumn{9}{|l|}{ Quality of life } \\
\hline SF-36 (mental health) ${ }^{2}$ & 53.97 & 5.63 & 48.49 & 8.97 & 48.99 & 8.00 & 44.87 & 10.33 \\
\hline SF-36 (physical health) ${ }^{a}$ & $\$ 1.93$ & 6.89 & $\$ 1.91$ & 8.29 & 48.80 & 9.22 & 46.53 & 10.95 \\
\hline Mean number of disability days ${ }^{b}$ during past 4 weeks & 0.56 & 2.19 & 1.34 & 4.40 & 1.47 & 4.21 & 3.44 & 7.15 \\
\hline \multicolumn{9}{|l|}{ During past 12 months, mesn number of } \\
\hline General practice visits & 1.83 & 2.90 & 3.11 & 4.58 & 3.22 & 4.96 & 4.12 & 7.16 \\
\hline Specialis visits & 3.08 & 5.75 & 5.06 & 8.09 & 6.08 & 9.85 & 8.41 & 11.22 \\
\hline Days in hospital & 0.83 & 4.96 & 1.50 & 6.68 & 1.45 & 8.34 & 2.33 & 8.16 \\
\hline Substance use during past 12 months & $n$ & $\%$ & $n$ & $\%$ & $n$ & $\%$ & $n$ & $\%$ \\
\hline Harmful alcohol use & 112 & 15.22 & 346 & 19.6 & 199 & 18.27 & 105 & 18.39 \\
\hline Daily smoking (over at least 4 woeks) & 237 & 31.67 & 613 & 32.56 & 341 & 32.37 & 194 & 34.82 \\
\hline Any illicit medication/substance use & 43 & 5.05 & 135 & 6.94 & 87 & 9.09 & 67 & 12.14 \\
\hline
\end{tabular}

no $P S$ no significant pain symptoms, EPS explained pain symptoms, UPS unexplained pain symptons, $P D$ pain disorder, $M$ mean (weighted), $S D$ standard deviation (weighted), $n$ number (unweighted), \% percent (weighed)

" SF-36 sum scores mental and physical health $(\mathrm{MW}=50, \mathrm{SD}=10)$

b Days being unable to carry out usual activities 


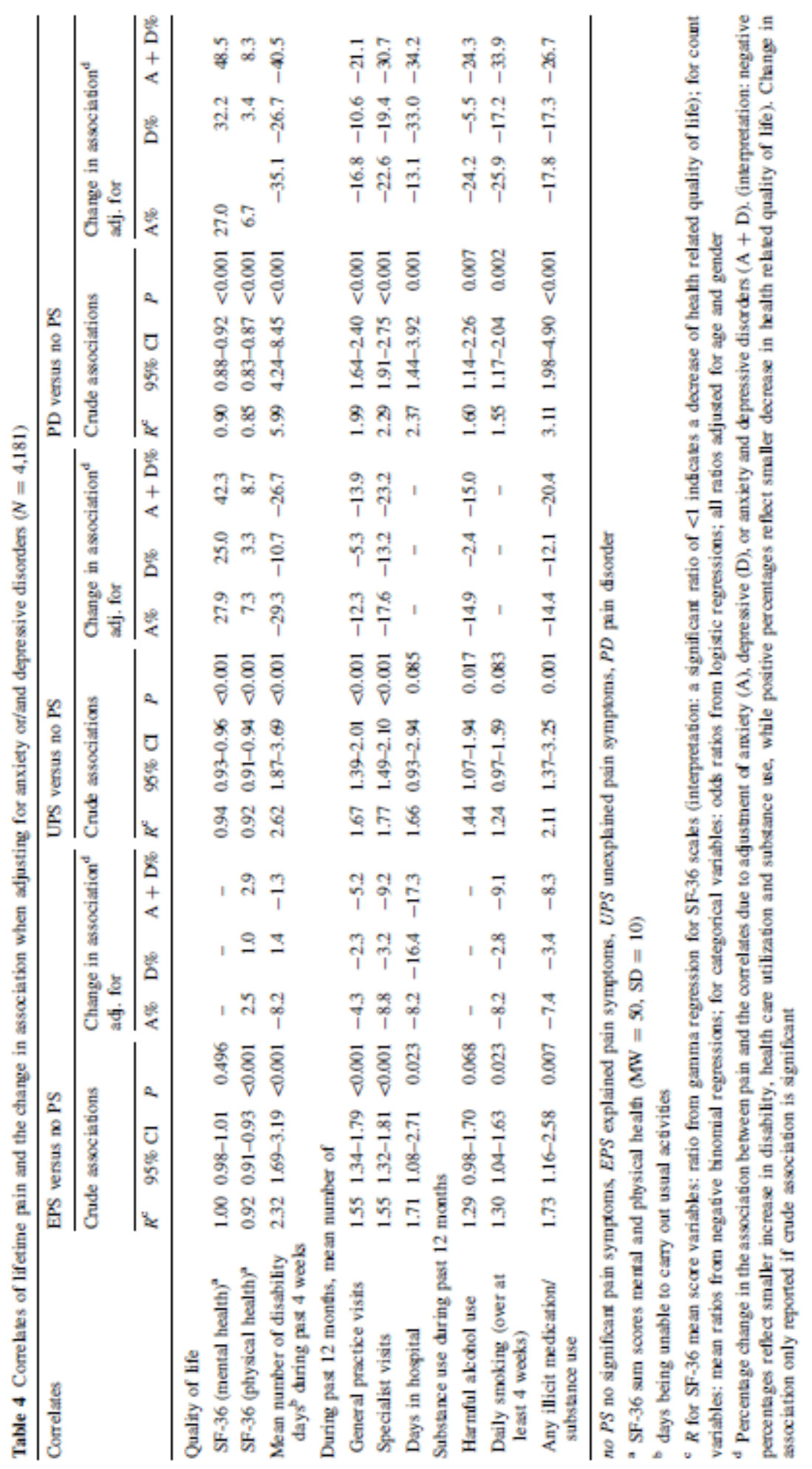

\section{References}

1. Alonso J, Angermeyer MC, Bernert S, Bruffaerts R, Brugha TS, Bryson H, de Girolamo G, de Graaf R, Demyttenaere K, Gasquet I, Haro JM, Katz SJ, Kessler RC, Kovess V, Lepine JP, Ormel J, Polidori G, Russo LJ, Vilagut G, Almansa J, Arbabzadeh-Bouchez S, Autonell J, Bernal M, BuistBouwman MA, Codony M, Domingo-Salvani A, Ferrer M, Joo SS, Martinez-Alonso M, Matschinger H, Mazzi F, Morgan Z, Morosini P, Palacin C, Romera B, Taub N, Vollebergh WAM (2004) 
Disability and quality of life impact of mental disorders Europe: results from the European Study of the Epidemiology of Mental Disorders (ESEMeD) project. Acta Psychiatr Scand 109:38-46 2. Andlin-Sobocki P, Jönsson B, Wittchen HU, Olesen J (2005) Cost of disorders of the brain in Europe. Eur J Neurol 12:1-27

3. APA (1987) Diagnostic and statistical manual of mental disorders. American Psychiatric Press, Washington

4. APA (1994) Diagnostic and statistical manual of mental disorders. American Psychiatric Press, Washington

5. APA (2000) Diagnostic and statistical manual of mental disorders, text revision, 4th edn. American Psychiatric Press, Washington

6. Asmundson GJG, Norton PJ, Vlaeyen JWS (2004) Fear-avoidance models of chronic pain: an overview. In: Asmundson GJG, Vlaeyen JWS, Crombez G (eds) Understanding and treating fear of pain. Oxford University Press, Oxford, pp 3-24

7. Bair MJ, Robinson RL, Eckert GJ, Stang PE, Croghan TW, Kroenke K (2004) Impact of pain on depression treatment response in primary care. Psychosom Med 66:17-22

8. Bair MJ, Robinson RL, Katon W, Kroenke K (2003) Depression and pain comorbidity - a literature review. Arch Int Med 163:2433-2445

9. Banks SM, Kerns RD (1996) Explaining high rates of depression in chronic pain: a diathesis-stress framework. Psychol Bull 119:95-110

10. Bao YH, Sturm R, Croghan TW (2003) A national study of the effect of chronic pain on the use of health care by depressed persons. Psychiatr Serv 54:693-697

11. Beesdo K, Hoyer J, Jacobi F, Low NCP, Höfler M, Wittchen HU (2009) Association between generalized anxiety levels and pain in a community sample: evidence for diagnostic specificity. $\mathrm{J}$ Anxiety Disord. doi: 10.1016/j.janxdis.2009.02.007

12. Blackburn-Munro G, Blackburn-Munro RE (2001) Chronic pain, chronic stress and depression: coincidence or consequence? J Neuroendocrinol 13:1009-1023

13. Bullinger M, Alonso J, Apolone G, Leplège A, Sullivan M, Wood-Dauphinee S, Gandek B, Wagner A, Aaronson N, Bech P, Fukuhara S, Kaasa S, WareJr JE (1998) Translating health status questionnaires and evaluating their quality. The IQOLA project approach. J Clin Epidemiol 51:913923

14. Bullinger M, Kirchberger I (1998) SF-36 Fragebogen zum Gesundheitszustand. Handanweisung. Hogrefe, Göttingen

15. Butterworth P, Crosier T (2004) The validity of the SF-36 in an Australian National Household Survey: demonstrating the applicability of the Household Income and Labour Dynamics in Australia (HILDA) Survey to examination of health inequalities. BMC Public Health 4:44

16. Campbell LC, Clauw DJ, Keefe FJ (2003) Persistent pain and depression: a biopsychosocial perspective. Biol Psychiatry 54:399-409

17. Cohen S, Rodriguez MS (1995) Pathways linking affective disturbances and physical disorders. Health Psychol 14:374-380

18. Currie SR, Wang J (2004) Chronic back pain and major depression in the general Canadian population. Pain 107:54-60

19. de Waal MWM, Arnold IA, Spinhoven P, Eekhof JAH, Assendelft WJJ, van Hemert AM (2009)

The role of comorbidity in the detection of psychiatric disorders with checklists for mental and physical symptoms in primary care. Soc Psychiatry Psychiatr Epidemiol 44:78-85

20. Demyttenaere K, Bonnewyn A, Bruffaerts R, Brugha T, De Graaf R, Alonso J (2006) Comorbid painful physical symptoms and depression: prevalence, work loss, and help seeking. J Affect Disord 92:185-193

21. Demyttenaere K, Bruffaerts R, Lee S, Posada-Villa J, Kovess V, Angermeyer MC, Levinson D, de Girolamo G, Nakane H, Mneimneh Z, Lara C, de Graaf R, Scott KM, Gureje O, Stein DJ, Haro JM, Bromet EJ, Kessler RC, Alonso J, Von Korff M (2007) Mental disorders among persons with chronic back or neck pain: results from the World Mental Health Surveys. Pain 129:332-342

22. Dersh J, Polatin PB, Gatchel RJ (2002) Chronic pain and psychopathology: research findings and theoretical considerations. Psychosom Med 64:773-786

23. Ellert U, Bellach B-M (1999) Der SF-36 im Bundes-Gesundhetissurvey - Beschreibung einer aktuellen Normstichprobe. Gesundheitswesen 61:S184-S190 
24. Eriksen J, Jensen MK, Sjogren P, Ekholm O, Rasmussen NK (2003) Epidemiology of chronic nonmalignant pain in Denmark. Pain 106:221-228

25. Fishbain DA, Cutler R, Rosomoff HL, Rosomoff RS (1997) Chronic pain-associated depression: antecedent or consequence of chronic pain? A review. Clin J Pain 13:116-137

26. Fröhlich C, Jacobi F, Wittchen H-U (2006) DSM-IV pain disorder in the general population - an exploration of the structure and threshold of medically unexplained pain symptoms. Eur Arch Psychiatry Clin Neurosci 256:187-196

27. Geerlings SW, Twisk JWR, Beekman ATF, Deeg DJH, Van Tilburg W (2002) Longitudinal relationship between pain and depression in older adults: sex, age and physical disability. Soc Psychiatry Psychiatr Epidemiol 37:23-30

28. Gonzalez VA, Martelli MF, Baker JM (2000) Psychological assessment of person with chronic pain. ***NeuroRehabil 14:69-83

29. Grabe HJ, Baumeister SE, John U, Freyberger HJ, Völzke H (2009) Association of mental distress with health care utilization and costs: a 5-year observation in a general population. Soc Psychiatry Psychiatr Epidemiol. doi: 10.1007/s001327-009-0005-9

30. Grabe HJ, Meyer C, Hapke U, Rumpf H-J, Freyberger HJ, Dilling H, John U (2003) Somatoform pain disorder in the general population. Psychother Psychosom 72:88-94

31. Grothe DR, Scheckner B, Albano D (2004) Treatment of pain syndromes with venlafaxine.

Pharmacotherapy 24:621-629

32. Gureje O, Simon GE, Von Korff M (2001) A cross-national study of the course of persistent pain in primary care. Pain 92:195-200

33. Gureje O, Von Korff M, Kola L, Demyttenaere K, He Y, Posada-Villa J, Lepine JP, Angermeyer MC, Lewinson D, de Girolamo G, Iwata N, Karam A, Borges GLG, De Graaf R, Browne MO, Stein DJ, Haro JM, Bromet EJ, Kessler RC, Alonso J (2008) The relation between multiple pains and mental disorders: results from the World Mental Health Surveys. Pain 135:82-91

34. Gureje O, Von Korff M, Simon GE, Gater R (1998) Persistent pain and well-being. A World Health Organization study in primary care. J Am Med Assoc 280:147-151 